\title{
Bp stars in Orion OB1 association
}

\author{
Iosif I. Romanyuk ${ }^{1}$ and Ilya A. Yakunin ${ }^{2}$ \\ ${ }^{1}$ Special Astrophysical Observatory of Russian Academy of Sciences, \\ 369167, Nizhny Arkhyz, Russia \\ email: roman@sao.ru \\ ${ }^{2}$ Special Astrophysical Observatory of Russian Academy of Sciences, \\ 369167, Nizhny Arkhyz, Russia \\ email: elias@sao.ru
}

\begin{abstract}
A total of $85 \mathrm{CP}$ stars of various types are identified among 814 members of the Orion OB1 association. We selected 59 Bp stars, which account for $13.4 \%$ of the total number of B type stars in the association. The fraction of peculiar B type stars in the association is found to be twice higher than that of peculiar A type stars.

Magnetic field are found in 22 stars, 17 of them are objects with anomalous helium lines. No significant differences are found between the field strengths in the Bp type stars of the association and Bp type field stars. We identified 17 binaries, which make up $20 \%$ of the total number of peculiar stars studied which is the standard ratio for CP stars.
\end{abstract}

Keywords. stars:chemically peculiar-open clusters and associations:individual:Orion OB1

\section{Introduction}

The Orion constellation hosts one of the most popular groups of early-type stars in the solar neighborhood - the Orion OB1 association. Blaau (1964) identified four regions inside the association - subgroups: a (corresponds to the northen part), b (the Orion's Belt), c (the region located south of Orion's Belt), and d (the very compact region located in the central part of the association) that slightly differ in age and stellar composition.

Most of the objects in the Orion OB1 association are normal hot main sequence stars; however, the association also includes pre-MS objects, like HAEBE stars, T Tau-type stars, and various anomalous (peculiar) stars. Chemically peculiar (CP) stars differ from normal stars by their anomalous chemical composition which shows up in enhanced or weakened intensity of lines of certain elements in the stellar spectrum.

Renson and Manfroid (2009) published the most detailed catalog of CP stars, which includes more than 8200 objects. Over the past quarter-century many new observations of CP stars have been performed. Our aim is to thoroughly analyze massive chemically peculiar and magnetic stars in the Ori OB1 association using all available data. For a review and analysis of the main studies on this subject, see Romanyuk \& Yakunin (2012) and Romanyuk et al., (2013).

\section{CP stars in the association}

Groups of hot stars in the Ori OB1 association have repeatedly attracted the researcher's attention. Here we consider only the issues related to chemically peculiar stars and magnetic field of these objects.

Borra \& Landstreet (1979) discovered very strong magnetic fields in a group of B-type stars with enhanced helium lines in young clusters in Orion. Klochkova (1985) performed spectroscopic observations of $24 \mathrm{CP}$ stars to determine the distance moduli and ages of 
Table 1. Age of subgroups and number of normal and CP stars

\begin{tabular}{|c|c|c|c|c|}
\hline subgroup & age, $\log \mathrm{t}$ & all stars & $\mathrm{CP}$ stars & fraction, $\%$ \\
\hline Ori OB1a & 7.05 & 311 & 24 & 7.7 \\
Ori OB1b & 6.23 & 139 & 21 & 15.1 \\
Ori OB1c & 6.66 & 350 & 37 & 10.6 \\
Ori OB1d & 6.0 & 14 & 3 & 21.4 \\
\hline
\end{tabular}

Table 2. Number of CP stars in different subroups

\begin{tabular}{|l|c|c|c|c|c|}
\hline Peculiarity type & total & Ori OB1a & Ori OB1b & Ori OB1c & Ori OB1d \\
\hline Am & 23 & 6 & 4 & 13 & 0 \\
He-strong & 7 & 1 & 3 & 1 & 2 \\
He-weak & 27 & 7 & 8 & 12 & 0 \\
Si, Si+ & 19 & 6 & 4 & 8 & 0 \\
Other & 9 & 3 & 1 & 4 & 1 \\
\hline
\end{tabular}

subroups. Brown et al. (1994) reported the results of photometric observations in the Walraven system for 814 stars, for all identified of suspected association members. They determined the effective temperatures, surface gravities, luminosities and masses for all 814 stars. They also detemined the distance moduli and showed that the near and far edges of clouds in the Orion OB1 association are located at the distances of about 320 and 500 pc, respectively. We decided to identify chemically peculiar stars among the 814 objects of this list. We consider a star to be peculiar if it is appeares in the catalog by Renson and Manfroid (2009).

We selected $85 \mathrm{CP}$ stars in the direction of the Ori OB1 association. We list these objects in paper by Romanyuk et al., (2013). Most of them (59 objects) are Bp stars, however, we also found $23 \mathrm{Am}$ and 3 Ap stars. We performed magnetic field observations using the $6 \mathrm{~m}$ Russian telescope.

The age of subgroups, number of normal and CP stars in each subgroup and fraction of CP stars are presented in Table 1.

We determine distances for most of the stars closer than $250 \mathrm{pc}$ from their Hipparcos parallaxes and we estimate the distances to more distant objects from their temperatures and luminosities. Proper motions have been measured for all CP stars.

The sample of peculiar stars is offset relative to the entire sample both in terms of temperature and luminosity. The fraction of hot stars is greater among peculiar stars. The effective-temperature distribution for the entire sample and CP stars peak at $\log T_{e}=3.95$ and $\log T_{e}=4.15$ (for details see Romanyuk et al., (2013). The distribution of CP stars in different subroups is presented in Table 2.

The catalog of CP stars by Renson and Manfroid (2009) includes 23 Am stars in the directions of the association. This is surprising given that low-mass Am stars should not have yet evolved enough to settle onto the main sequence. We therefore decided to verify whether the Am stars in question are foreground objects and not members of the association. Parallaxes are available for 14 of the 23 Am stars and they support conclusively the above hypothesis - these objects are located closer than $300 \mathrm{pc}$. The distances of remaining nine stars can be determined only from analysis of their tempearures and luminosities. The result of analysis is the same: the distances to Am stars are closer than $300 \mathrm{pc}$.

Occurence frequency of CP stars.

The fraction of CP stars can be seen to be smallest (7.7\%) in the oldest subgroup (a) of the association. It is twice higher $(15.1 \%)$ in the substantially younger subgroup (b). The fraction of peculiar stars is even higher in the youngest subgroup (d), however, it 


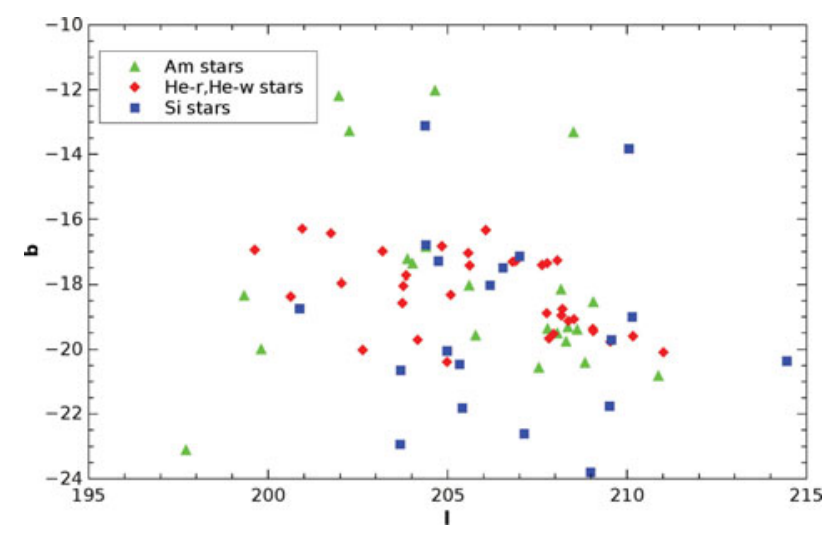

Figure 1. Spatial distribution of CP stars in the Ori OB1 assocation.

contains too few (only 14) objects to allow any statistical conclusions. The lists of Brown et al.(1994) contains 661 stars in the two older groups (a) and (c): $81.2 \%$ of the total number of stars in the association. We adopt this fraction as standard. Bp stars are much less concentrated in old subroups. Thus the fraction of He-weak stars in the old subgroups (a) and (c) is equal to $70 \%$; that of Si-stars, to $52.6 \%$, and that of He-strong stars, to $28.6 \%$. The discrepancies are quite substantial and significant. The predominance of stars with enhanced helium lines is especially conspicuous in the young subgroups (b) and (d). It is interesting that the fraction of He-strong stars in subgroups (b) and (d), whose ages do not exceed $2 \mathrm{Myr}$, is three times higher than that of He-weak stars in the same subgroups.

Spatial distribution of CP stars.

Spatial distribution of CP stars in the Orion OB1 association is demonstrated on Fig. 1.

The group of Am stars is located separately from the other objects and closer to us. It can be concluded that Am stars are foreground objects which are merely seen projected against the association. Stars with anomalous helium lines belongs to cluster and mostly concentrate in subgroups (b) and (c) -20 objects, whereas subgroup (a) contains only 8 objects. Silicon stars concentrate mostly in subgroups (a) and (c) -14 out of 18 objects.

Binary CP stars.

We identified 17 physical binaries among $85 \mathrm{CP}$ stars of the association, which makes up a typical fraction of $20 \%$. These stars are distributed by peculiarity type as follows: He-strong -5 (out of 7 ). He-weak -4 (out of 27 ), $\mathrm{SiSi}+-2$ (out of 10 ), $\mathrm{Am}-1$ (out of 23 ), and other types -5 (out of 9 ).

The largest fraction of binaries is found among He-strong stars. The two stars with no companion found are HD 36982 and HD 37776. Weak Am stars are very poorly studied, and abnormally low number of binaries may be result of absence of radial velocity measurements. He-weak and $\mathrm{SiSi}+$ stars show the fraction of binaries lower than normal.

\section{Magnetic fields}

We found 22 magnetic stars in the association of which 21 are Bp stars. Table 3 lists the stars with reliably detected magnetic field.

Eight of the magnetic stars are binary (36.4\%). The overwhelming majority (17 out of 22 , or $77 \%$ ) are objects with anomalous helium lines. The fraction of magnetic stars in the inner subgroup (b) is twice higher than in the outer subroups (a) and (c). 
Table 3. Magnetic stars in the Ori OB1 association

\begin{tabular}{l|l|c||l|l|l|}
\hline Star & Sp Pec & $B_{e}$ extrema & Star & Sp Pec & $B_{e}$ extrema \\
\hline HD 35008 & B8 Si & -340 & $\mid$ HD 36668 & B7 He-wk, Si & $-2200 /+2000$ \\
HD 35298 & B6 He-wk & $-3000 /+3000$ & HD 36916 & B8 He-wk, Si & $-1100 / 0$ \\
HD 35456 & B7 He-wk & $-400 /+1080$ & HD 36955 & A2 CrEu & $-1300 /-410$ \\
HD 35502 & B6 SrCrSi & $-2250 /-180$ & HD 37017 & B2 He-strong & $-2300 /-300$ \\
HD 35730 & B7 He-wk & $-450 /+250$ & HD 37058 & B2 He-wk, Sr & $-1200 /+1200$ \\
HD 36313 & B8 He-wk & $-1500 /-1100$ & HD 37140 & B8 SiSr & $-1050 /+400$ \\
HD 36429 & B6 He-wk & $-840 /+160$ & HD 37479 & B2 He-strong & $-1600 /+3500$ \\
HD 36485 & B2 He-strong & $-3700 /+3000$ & HD 37642 & B9 He-wk, Si & $-3000 /+3000$ \\
HD 36526 & B8 He-wk,Si & $-3500 /+3400$ & HD 37687 & B7 He-wk & $-600 /+500$ \\
HD 36540 & B7 He-wk & $-900 /+1030$ & HD 37776 & B2 He-strong & $-2000 /+2000$ \\
HD 36629 & B3 He-wk & $-1300 /+1100$ & HD 290665 & B9 SrCrEu & $-1600 /+5000$ \\
\hline
\end{tabular}

We see no significant differences between magnetic stars of the Ori OB1 association and Bp stars in general in terms of magnetic field strength. However, despite poor statistics, He-strong stars can be seen to posses, on the whole, a factor of $1.5-2$ stronger fields than He-wk stars.

\section{Conclusion}

We thus identified $85 \mathrm{CP}$ stars in the direction toward the young Ori OB1 association. Our CP stars are distributed by peculiarity types as follows: 23 Am stars, 3 Ap stars and 59 Bp stars. The fraction of peculiar B-type stars in the association is twice higher than the corresponding fraction of peculiar A-type stars. The association includes 22 magnetic stars; 21 of them are Bp stars, and only one is an Ap star. We suggest that when the stars were born in the Orion OB1 association the magnetic fields formed mostly in the objects that later developed helium rather than silicon anomalies.

\section{References}

Blaau A., 1964, ARAA, 2, 236.

Romanyuk I. I. \& Yakunin I. A., 2012, Astrophys Bull., 67, 177.

Romanyuk I. I. et al., 2013, Astrophys Bull., 68, 300.

Borra E. F. \& Landstreet J. D., 1979, A\&GA, 228, 809.

Klochkova V. G., 1985, PAZ, 11,209.

Brown A. G. A. et al., 1994, A\&A, 289, 101.

Renson P. \& Manfroid J., 2009, A\&\&A, 498, 961. 